\title{
Digital Expansions Endowed with Fixed Point Theory
}

\author{
Kumari Jyoti, Asha Rani* \\ Department of Mathematics, SRM University, Haryana, Sonepat- 131001, India \\ *Corresponding author: asha.dahiya27@gmail.com, asha.dahiya1509@gmail.com
}

Received March 14, 2017; Revised June 20, 2017; Accepted July 11, 2017

\begin{abstract}
The area of fixed point theory is very active in many branches of mathematics and other related disciplines such as image processing, computer vision, applied mathematics, etc. The main goal in this theory is to solve many problems and to give some useful applications. The aim of this paper is to associate fixed point theory and digital images.
\end{abstract}

Keywords: expansive mappings, digital- $\alpha-\psi$ - expansive mappings, fixed point theorems, digital metric space

Cite This Article: Kumari Jyoti, and Asha Rani, "Digital Expansions Endowed with Fixed Point Theory.” Turkish Journal of Analysis and Number Theory, vol. 5, no. 5 (2017): 146-152. doi: 10.12691/tjant-5-5-1.

\section{Introduction}

The dawn of the fixed point theory starts when in 1912 Brouwer [1] proved a fixed point result for continuous self maps on a closed ball. In the last few decades, fixed point theory has been one of the most interesting research fields in nonlinear functional analysis. In 1984 Wang [21] introduced the expansive mappings and proved fixed point results for them.

Digital topology is a developing area which is related to features of 2D and 3D digital images using general topology and functional analysis. Up to now, several developments have occurred in the study of digital topology. Digital topology was first studied by Rosenfield [19]. Kong [18], then introduced the digital fundamental group of a discrete object. Boxer [2] has given the digital versions of several notions from topology and [3] studied a variety of digital continuous functions. Some results and characteristic properties on the digital homology groups of 2D digital images are given in [8] and [17]. Ege and Karaca $[9,10]$ give relative and reduced Lefschetz fixed point theorem for digital images. They also calculate degree of antipodal map for the sphere like digital images using fixed point properties. In [12,13,14,15] Ege and Karaca studied some more properties of related to digital topologies. Boxer et. al. [7] gave approximate digital fixed points and universal functions for digital metric spaces. Ege and Karaca [11] then defined a digital metric space and proved the famous Banach Contraction Principle for digital images.

\section{Preliminaries}

Let $X$ be a subset of $\mathbb{Z}^{n}$ for a positive integer $n$ where $\mathbb{Z}^{n}$ is the set of lattice points in the $n$-dimensional Euclidean space and $\rho$ represent an adjacency relation for the members of $X$. A digital image consists of $(X, \rho)$.
Definition 2.1 [4]: Let $l, n$ be positive integers, $1 \leq l \leq n$ and two distinct points

$$
a=\left(a_{1}, a_{2}, \ldots, a_{n}\right), b=\left(b_{1}, b_{2}, \ldots, b_{n}\right) \in \mathbb{Z}^{n}
$$

$a$ and $b$ are $k_{l}$ - adjacent if there are at most $l$ indices $i$ such that $\left|a_{i}-b_{i}\right|=1$ and for all other indices $j$ such that $\left|a_{j}-b_{j}\right| \neq 1, a_{j}=b_{j}$.

There are some statements which can be obtained from definition 2.1:

- $\quad a$ and $b$ are 2- adjacent if $|a-b|=1$.

- $a$ and $b$ in $\mathbb{Z}^{2}$ are 8- adjacent if they are distinct and differ by at most 1 in each coordinate.

- $a$ and $b$ in $\mathbb{Z}^{3}$ are 26- adjacent if they are distinct and differ at most 1 in each coordinate.

- $\quad a$ and $b$ in $\mathbb{Z}^{3}$ are 18 - adjacent if are 26- adjacent and differ by at most two coordinates.

- $\quad a$ and $b$ are 6- adjacent if they are 18- adjacent and differ in exactly one coordinate.

A $\rho$-neighbour [4] of $a \in \mathbb{Z}^{n}$ is a point of $\mathbb{Z}^{n}$ that is $\rho$ - adjacent to $a$ where $\rho \in\{2,4,8,6,18,26\}$ and $n \in 1,2,3$. The set

$$
\mathrm{N}_{\rho}(a)=\{b \mid b \text { is } \rho \text {-adjacent to } a\}
$$

is called the $\rho$ - neighbourhood of $a$. A digital interval [9] is defined by

$$
[p, q]_{\mathbb{Z}}=\{z \in \mathbb{Z} \mid p \leq z \leq q\}
$$

where $p, q \in \mathbb{Z}$ and $p<q$.

A digital image $X \subset \mathbb{Z}^{n}$ is $\rho$ - connected [16] if and only if for every pair of different points $u, v \in X$, there is a set $\left\{u_{0}, u_{1}, \ldots, u_{r}\right\}$ of points of digital image $X$ such that $u=u_{0}, v=u_{r}$ and $u_{i}$ and $u_{i+1}$ are $\rho$ - neighbours where $i=0,1, \ldots, r-1$.

Definition 2.2: Let $\left(X, \rho_{0}\right) \subset \mathbb{Z}^{n_{0}},\left(Y, \rho_{1}\right) \subset \mathbb{Z}^{n_{1}}$ be digital images and $T: X \rightarrow Y$ be a function.

- $T$ is said to be $\left(\rho_{0}, \rho_{1}\right)$ - continuous [4], if for all $\rho_{0}$ connected subset $E$ of $X, f(E)$ is a $\rho_{1}$ - connected subset of $Y$. 
- For all $\rho_{0}$ - adjacent points $\left\{u_{0}, u_{1}\right\}$ of $X$, either $T\left(u_{0}\right)=T\left(u_{1}\right)$ or $T\left(u_{0}\right)$ and $T\left(u_{1}\right)$ are a $\rho_{1}$ - adjacent in $Y$ if and only if $T$ is $\left(\rho_{0}, \rho_{1}\right)$ continuous [4].

- If $f$ is $\left(\rho_{0}, \rho_{1}\right)$ - continuous, bijective and $T^{-1}$ is $\left(\rho_{1}, \rho_{0}\right)$ - continuous, then $T$ is called $\left(\rho_{0}, \rho_{1}\right)$ isomorphism [5] and denoted by $X \cong{ }_{\left(\rho_{0}, \rho_{1}\right)} Y$.

A $(2, \rho)$ - continuous function $T$, is called a digital $\rho$ - path [4] from $u$ to $v$ in a digital image $X$ if $T:[0, m]_{\mathbb{Z}} \rightarrow$ $X$ such that $T(0)=u$ and $T(m)=v$. A simple closed $\rho$ - curve of $m \geq 4$ points [4] in a digital image $X$ is a sequence $\{T(0), T(1), \ldots, T(m-1)\}$ of images of the $\rho$ - path $T:[0, m-1]_{\mathbb{Z}} \rightarrow X$ such that $T(i)$ and $T(j)$ are $\rho$-adjacent if and only if $j=i \pm \bmod m$.

Definition 2.5 [11]: A sequence $\left\{x_{n}\right\}$ of points of a digital metric space $(X, d, \rho)$ is a Cauchy sequence if for all $\in>0$, there exists $\delta \in \mathbb{N}$ such that for all $n, m>\delta$, then

$$
d\left(x_{n}, x_{m}\right)<\in \text {. }
$$

Definition 2.6 [11]: A sequence $\left\{x_{n}\right\}$ of points of a digital metric space $(X, d, \rho)$ converges to a limit $p \in X$ if for all $\in>0$, there exists $\alpha \in \mathbb{N}$ such that for all $n>\delta$, then

$$
d\left(x_{n}, p\right)<\in \text {. }
$$

Definition 2.7 [11]: A digital metric space $(X, d, \rho)$ is a digital metric space if any Cauchy sequence $\left\{x_{n}\right\}$ of points of $(X, d, \rho)$ converges to a point $p$ of $(X, d, \rho)$.

Definition 2.8 [11]: Let $(X, \rho)$ be any digital image. A function $T:(X, \rho) \rightarrow(X, \rho)$ is called right- continuous if $f(p)=\lim _{x \rightarrow p^{+}} T(x)$ where, $p \in X$.

Definition 2.9 [11]: Let, $(X, d, \rho)$ be any digital metric space and $T:(X, d, \rho) \rightarrow(X, d, \rho)$ be a self digital map. If there exists $\alpha \in(0,1)$ such that for all $x, y \in X$,

$$
d(f(x), f(y)) \leq \alpha d(x, y),
$$

then $T$ is called a digital contraction map.

Proposition 2.10 [11]: Every digital contraction map is digitally continuous.

Theorem 2.11 [11]: (Banach Contraction principle) Let $(X, d, \rho)$ be a complete metric space which has a usual Euclidean metric in $\mathbb{Z}^{n}$. Let, $T: X \rightarrow X$ be a digital contraction map. Then $T$ has a unique fixed point, i.e. there exists a unique $p \in X$ such that $f(p)=p$.

In order to introduce digital- $\alpha-\psi$-expansive mappings, we use the following definition, given by Samet et. al. [20]:

Definition 2.16 [20] Let $\Psi$ denote the family of all functions $\psi:[0, \infty) \rightarrow[0, \infty)$ which satisfy the following :

(i) $\psi^{n}(t)<\infty$ for each $t>0$, where $\psi^{n}$ is the nth iterate of $\psi$;

(ii) $\psi$ is non-decreasing.

\section{Main Results}

\subsection{Expansive Maps}

Definition 3.1: Suppose that $(X, d, \rho)$ is a complete digital metric space and $T: X \rightarrow X$ is any mapping. The mapping
$T$ satisfy the condition $d(T(x), T(y)) \geq k d(x, y)$ holds for all $x, y \in X$ and $k>1$, then $T$ is called a digital expansive mapping.

Theorem 3.2: Let $T$ be a digital expansive mapping on a complete digital metric space $X$, and $T$ is onto. Then $T$ has a fixed point in $X$.

Proof: Let, $x_{0} \in X$, since $T$ is onto, there exists an element $x_{1}$ satisfying $x_{1} \in T^{-1}\left(x_{0}\right)$. By the same way, we can choose, $x_{n} \in T^{-1}\left(x_{n-1}\right)$ where $(n=2,3,4, \ldots)$.

If $x_{m-1}=x_{m}$ for some $m$, then $x_{m}$ is a fixed point of $T$. Without loss of generality, we can suppose $x_{n} \neq x_{n-1}$ for every $n$. So,

$$
\begin{aligned}
& d\left(x_{n-1}, x_{n}\right)=d\left(T\left(x_{n}\right), T\left(x_{n+1}\right)\right) \geq k d\left(x_{n}, x_{n+1}\right) \\
& d\left(x_{n}, x_{n+1}\right) \leq\left(\frac{1}{k}\right) d\left(x_{n-1}, x_{n}\right) \leq\left(\frac{1}{k}\right)^{n} d\left(x_{0}, x_{1}\right) \\
& d\left(x_{n}, x_{n+m}\right) \leq d\left(x_{n}, x_{n+1}\right)+\ldots+d\left(x_{n+m-1}, x_{n+m}\right) \\
& \leq\left(\frac{1}{k}\right)^{n+1} d\left(x_{0}, x_{1}\right)+\ldots+\left(\frac{1}{k}\right)^{n+m} d\left(x_{0}, x_{1}\right) \\
& \leq \frac{\left(\frac{1}{k}\right)^{n+1}}{1-\frac{1}{k}} d\left(x_{0}, x_{1}\right) .
\end{aligned}
$$

Clearly, $\left\{x_{n}\right\}$ is a digital Cauchy sequence in $X$. Since, $X$ is complete $\left\{x_{n}\right\}$ converges to some $x \in X$. Since $T$ is onto there exists $y \in X$ such that $y \in T^{-1}(x)$ and for infinitely many $n, x_{n} \neq x$, for such $n$

$$
d\left(x_{n}, x\right)=d\left(T\left(x_{n+1}\right), T(y)\right) \geq k d\left(x_{n+1}, y\right) .
$$

On taking limit as $n \rightarrow \infty$, we get,

$$
0 \geq k \lim _{n \rightarrow \infty} d\left(x_{n+1}, y\right) \Rightarrow d(x, y) \geq 0 \Rightarrow x=y .
$$

Thus, $T$ has a fixed point in $X$.

Corollary 3.3: Let $T$ be a digital expansive mapping on a complete digital metric space $X$, and $T$ is bijective. Then $T$ has a unique fixed point in $X$.

Proof: Since, $T$ is bijective therefore, there is a unique element $y \in X$ for every $x \in X$ such that $y=T^{-1}(x)$. So, by obvious argument the fixed point is unique.

Theorem 3.4: Let, $(X, d, \rho)$ be a complete digital metric space and $T$ be an onto self map on $X$. Let $T$ satisfy the condition $\quad d(T(x), T(y)) \geq k\{d(x, T(x))+d(y, T(y))\}$ where $k \geq \frac{1}{2}$, then $T$ has a fixed point.

Proof: Let, $x_{0} \in X$, since $T$ is onto, there exists an element $x_{1}$ satisfying $x_{1} \in T^{-1}\left(x_{0}\right)$. By the same way, we can choose, $x_{n} \in T^{-1}\left(x_{n-1}\right)$ where $(n=2,3,4, \ldots)$.

If $x_{m-1}=x_{m}$ for some $m$, then $x_{m}$ is a fixed point of $T$. Without loss of generality, we can suppose $x_{n} \neq x_{n-1}$ for every $n$. So,

$$
\begin{aligned}
& d\left(x_{n-1}, x_{n}\right)=d\left(T\left(x_{n}\right), T\left(x_{n+1}\right)\right) \\
& \geq k\left\{d\left(x_{n}, T\left(x_{n}\right)\right)+d\left(x_{n+1}, T\left(x_{n+1}\right)\right)\right\} \\
& \geq 2 k d\left(x_{n+1}, x_{n}\right)
\end{aligned}
$$




$$
\begin{aligned}
& d\left(x_{n}, x_{n+1}\right) \leq\left(\frac{1}{2 k}\right) d\left(x_{n-1}, x_{n}\right) \leq\left(\frac{1}{2 k}\right)^{n} d\left(x_{0}, x_{1}\right) \\
& d\left(x_{n}, x_{n+m}\right) \leq d\left(x_{n}, x_{n+1}\right)+\ldots+d\left(x_{n+m-1}, x_{n+m}\right) \\
& \leq\left(\frac{1}{2 k}\right)^{n+1} d\left(x_{0}, x_{1}\right)+\ldots+\left(\frac{1}{2 k}\right)^{n+m} d\left(x_{0}, x_{1}\right) \\
& \leq \frac{\left(\frac{1}{2 k}\right)^{n+1}}{1-\frac{1}{2 k}} d\left(x_{0}, x_{1}\right) .
\end{aligned}
$$

Clearly, $\left\{x_{n}\right\}$ is a Cauchy sequence in $X$. Since, $X$ is complete $\left\{x_{n}\right\}$ converges to some $x \in X$. Since $T$ is onto there exists $y \in X$ such that $y \in T^{-1}(x)$ and for infinitely many $n, x_{n} \neq x$, for such $n$

$$
\begin{aligned}
& d\left(x_{n}, x\right)=d\left(T\left(x_{n+1}\right), T(y)\right) \\
& \geq k\left\{d\left(x_{n+1}, T\left(x_{n+1}\right)\right)+d(y, T(y))\right\} .
\end{aligned}
$$

On taking limit as $n \rightarrow \infty$, we get,

$$
0 \geq k d(y, T(y)) \Rightarrow d(y, x) \geq 0 \Rightarrow x=y \text {. }
$$

Thus, $T$ has a fixed point in $X$.

Theorem 3.5: Let, $(X, d, \rho)$ be a complete digital metric space and $T$ be an onto self map which is continuous on $X$. Let $T$ satisfy the condition

$$
d(T(x), T(y)) \geq k \mu
$$

where $k>1$, and

$$
\mu \equiv \mu(x, y) \in\left\{\begin{array}{l}
d(x, y), \frac{d(x, T(x))+d(y, T(y))}{2}, \\
\frac{\{d(x, T(y))+d(y, T(x))\}}{2}
\end{array}\right\}
$$

then $T$ has a fixed point.

Proof: Let, $x_{0} \in X$, since $T$ is onto, there exists an element $x_{1}$ satisfying $x_{1} \in T^{-1}\left(x_{0}\right)$. By the same way, we can choose, $x_{n} \in T^{-1}\left(x_{n-1}\right)$ where $(n=2,3,4, \ldots)$.

If $x_{m-1}=x_{m}$ for some $m$, then $x_{m}$ is a fixed point of $T$. Without loss of generality, we can suppose $x_{n} \neq x_{n-1}$ for every $n$. So,

$$
d\left(x_{n-1}, x_{n}\right)=d\left(T\left(x_{n}\right), T\left(x_{n+1}\right)\right) \geq k \mu_{n}
$$

where,

$$
\begin{aligned}
& \mu_{n} \in\left\{\begin{array}{l}
d\left(x_{n}, x_{n+1}\right), \frac{d\left(x_{n}, T\left(x_{n}\right)\right)+d\left(x_{n+1}, T\left(x_{n+1}\right)\right)}{2}, \\
\frac{d\left(x_{n}, T\left(x_{n+1}\right)\right)+d\left(x_{n+1}, T\left(x_{n}\right)\right)}{2}
\end{array}\right\} \\
& =\left\{\begin{array}{l}
d\left(x_{n}, x_{n+1}\right), \frac{d\left(x_{n}, x_{n-1}\right)+d\left(x_{n+1}, x_{n}\right)}{2}, \\
\frac{d\left(x_{n+1}, x_{n-1}\right)}{2}
\end{array}\right\} .
\end{aligned}
$$

Case 1: $d\left(x_{n-1}, x_{n}\right) \geq k d\left(x_{n}, x_{n+1}\right)$
Case 2: $d\left(x_{n-1}, x_{n}\right) \geq k \frac{d\left(x_{n}, x_{n-1}\right)+d\left(x_{n}, x_{n+1}\right)}{2}$

$$
\begin{gathered}
d\left(x_{n-1}, x_{n}\right)-\left(\frac{k}{2} d\left(x_{n-1}, x_{n}\right)\right) \geq \frac{k}{2} d\left(x_{n}, x_{n+1}\right), \\
\left(\frac{2-k}{k}\right) d\left(x_{n-1}, x_{n}\right) \geq d\left(x_{n}, x_{n+1}\right),
\end{gathered}
$$

$d\left(x_{n-1}, x_{n}\right) \geq \rho d\left(x_{n}, x_{n+1}\right)$ where $\rho=\frac{k}{2-k}>1$.

(since, if $1<k<1$, then clearly $\rho>1$ and if $k \geq 2$ then we get $d\left(x_{n}, x_{n+1}\right)<0$ which is a contradiction)

Case 3: $d\left(x_{n-1}, x_{n}\right) \geq k \frac{d\left(x_{n+1}, x_{n-1}\right)}{2}$, which is same as

Case2 since $d\left(x_{n+1}, x_{n-1}\right) \leq d\left(x_{n-1}, x_{n}\right)+d\left(x_{n}, x_{n+1}\right)$.

So, in each case $d\left(x_{n-1}, x_{n}\right) \geq \theta d\left(x_{n}, x_{n+1}\right)$ for some $\theta>1$.

$$
\begin{aligned}
& d\left(x_{n}, x_{n+m}\right) \leq d\left(x_{n}, x_{n+1}\right)+\ldots+d\left(x_{n+m-1}, x_{n+m}\right) \\
& \leq\left(\frac{1}{\theta}\right)^{n+1} d\left(x_{0}, x_{1}\right)+\ldots+\left(\frac{1}{\theta}\right)^{n+m} d\left(x_{0}, x_{1}\right) \\
& \leq \frac{\left(\frac{1}{\theta}\right)^{n+1}}{1-\frac{1}{\theta}} d\left(x_{0}, x_{1}\right) .
\end{aligned}
$$

Clearly, $\left\{x_{n}\right\}$ is a Cauchy sequence in $X$. Since, $X$ is complete $\left\{x_{n}\right\}$ converges to some $x \in X$. Since, $T$ is onto there exists $y \in X$ such that $y \in T^{-1}(x)$ and for infinitely many $n, x_{n} \neq x$, for such $n$

$$
d\left(x_{n}, x\right)=d\left(T\left(x_{n+1}\right), T(y)\right) \geq k \mu\left(x_{n+1}, y\right) .
$$

Now,

$$
\mu\left(x_{n+1}, y\right) \in\left\{\begin{array}{l}
d\left(x_{n+1}, y\right), \frac{d\left(x_{n+1}, T\left(x_{n+1}\right)\right)+d(y, T(y))}{2}, \\
\frac{d\left(x_{n+1}, T(y)\right)+d\left(y, T\left(x_{n+1}\right)\right)}{2}
\end{array}\right\} .
$$

On taking limit as $n \rightarrow \infty$, we get,

$$
\begin{aligned}
& \mu(x, y) \in\left\{\begin{array}{l}
d(x, y), \frac{d(x, T(x))+d(y, T(y))}{2}, \\
\left.\frac{d(x, T(y))+d(y, T(x))}{2}\right\}
\end{array}\right\} \\
& =\left\{d(x, y), \frac{d(x, T(x))+d(x, y)}{2}, \frac{d(y, T(x))}{2}\right\} .
\end{aligned}
$$

Also, $0 \geq k \mu(x, y)$, which implies $\mu(x, y)=0$. So, $d(x, y)=0$ or $d(y, T(x))=0$.

If $d(x, y)=0$, then $x=y$.

If $d(y, T(x))=0$, then $y=T(x)$ but $x=T(y)$ and $T$ is continuous. So, $x=y$.

(Since, by continuity of $T$, we get, $y=\lim _{n \rightarrow \infty} T\left(x_{n}\right)$. So $T\left(x_{n}\right)$ converges to $y$. Then, there exist $N \in \mathbb{N}$, such that, $\forall n>N, T\left(x_{n}\right) \in T^{-1}(x)$, by construction $x_{n+1} \in$ $T^{-1}(x)$ and hence by uniqueness of limit $x=y$ ) 
Thus, $T$ has a fixed point in $X$.

Theorem 3.6: Let, $(X, d, \rho)$ be a complete digital metric space and $T$ be an onto self map which is continuous on $X$. Let $T$ satisfy the condition

$$
d(T(x), T(y)) \geq k \mu
$$

where $k>1$, and

$$
\mu \equiv \mu(x, y) \in\left\{\begin{array}{l}
d(x, y), \frac{d(x, T(x))+d(y, T(y))}{2}, \\
d(x, T(y)), d(y, T(x))
\end{array}\right\}
$$

then $T$ has a fixed point.

Proof: Let, $x_{0} \in X$, since $T$ is onto, there exists an element $x_{1}$ satisfying $x_{1} \in T^{-1}\left(x_{0}\right)$. By the same way, we can choose, $x_{n} \in T^{-1}\left(x_{n-1}\right)$ where $(n=2,3,4, \ldots)$.

If $x_{m-1}=x_{m}$ for some $m$, then $x_{m}$ is a fixed point of $T$. Without loss of generality, we can suppose $x_{n} \neq x_{n-1}$ for every $n$. So,

$$
d\left(x_{n-1}, x_{n}\right)=d\left(T\left(x_{n}\right), T\left(x_{n+1}\right)\right) \geq k \mu_{n}
$$

where,

$$
\begin{aligned}
& \mu_{n} \in\left\{\begin{array}{l}
d\left(x_{n}, x_{n+1}\right), \frac{d\left(x_{n}, T\left(x_{n}\right)\right)+d\left(x_{n+1}, T\left(x_{n+1}\right)\right)}{2}, \\
d\left(x_{n}, T\left(x_{n+1}\right)\right), d\left(x_{n+1}, T\left(x_{n}\right)\right)
\end{array}\right\} \\
& =\left\{\begin{array}{l}
d\left(x_{n}, x_{n+1}\right), \frac{d\left(x_{n}, x_{n-1}\right)+d\left(x_{n+1}, x_{n}\right)}{2}, \\
d\left(x_{n+1}, x_{n-1}\right)
\end{array}\right\} .
\end{aligned}
$$

Case 1: $d\left(x_{n-1}, x_{n}\right) \geq k d\left(x_{n}, x_{n+1}\right)$

Case 2: $d\left(x_{n-1}, x_{n}\right) \geq k \frac{d\left(x_{n}, x_{n-1}\right)+d\left(x_{n}, x_{n+1}\right)}{2}$

$$
\begin{gathered}
d\left(x_{n-1}, x_{n}\right)-\left(\frac{k}{2} d\left(x_{n-1}, x_{n}\right)\right) \geq \frac{k}{2} d\left(x_{n}, x_{n+1}\right), \\
\left(\frac{2-k}{k}\right) d\left(x_{n-1}, x_{n}\right) \geq d\left(x_{n}, x_{n+1}\right),
\end{gathered}
$$

$d\left(x_{n-1}, x_{n}\right) \geq \rho d\left(x_{n}, x_{n+1}\right)$ where $\rho=\frac{k}{2-k}>1$

(since, if $1<k<2$, then clearly $\rho>1$ and if $k \geq 2$ then we get $d\left(x_{n}, x_{n+1}\right)<0$ which is a contradiction)

Case 3: $d\left(x_{n-1}, x_{n}\right) \geq k d\left(x_{n+1}, x_{n-1}\right)$, which is same Case 2 as $d\left(x_{n+1}, x_{n-1}\right) \leq d\left(x_{n-1}, x_{n}\right)+d\left(x_{n}, x_{n+1}\right)$

So, in each case $d\left(x_{n-1}, x_{n}\right) \geq \theta d\left(x_{n}, x_{n+1}\right)$ for some $\theta>1$.

$$
\begin{aligned}
& d\left(x_{n}, x_{n+m}\right) \leq d\left(x_{n}, x_{n+1}\right)+\ldots+d\left(x_{n+m-1}, x_{n+m}\right) \\
& \leq\left(\frac{1}{\theta}\right)^{n+1} d\left(x_{0}, x_{1}\right)+\ldots+\left(\frac{1}{\theta}\right)^{n+m} d\left(x_{0}, x_{1}\right) \\
& \leq \frac{\left(\frac{1}{\theta}\right)^{n+1}}{1-\frac{1}{\theta}} d\left(x_{0}, x_{1}\right) .
\end{aligned}
$$

Clearly, $\left\{x_{n}\right\}$ is a Cauchy sequence in $X$. Since, $X$ is complete $\left\{x_{n}\right\}$ converges to some $x \in X$. Since $T$ is onto there exists $y \in X$ such that $y \in T^{-1}(x)$ and for infinitely many $n, x_{n} \neq x$, for such $n$

$$
d\left(x_{n}, x\right)=d\left(T\left(x_{n+1}\right), T(y)\right) \geq k \mu\left(x_{n+1}, y\right) .
$$

Now,

$\mu\left(x_{n+1}, y\right) \in\left\{\begin{array}{l}d\left(x_{n+1}, y\right), \frac{d\left(x_{n+1}, T\left(x_{n+1}\right)\right)+d(y, T(y))}{2}, \\ d\left(x_{n+1}, T(y)\right), d\left(y, T\left(x_{n+1}\right)\right)\end{array}\right\}$.

On taking limit as $n \rightarrow \infty$, we get,

$$
\begin{aligned}
& \mu(x, y) \in\left\{\begin{array}{l}
d(x, y), \frac{d(x, T(x))+d(y, T(y))}{2}, \\
d(x, x), d(y, T(x))
\end{array}\right\} \\
& =\left\{d(x, y), \frac{d(x, T(x))+d(x, y)}{2}, d(y, T(x))\right\} .
\end{aligned}
$$

Also, $0 \geq k \mu(x, y)$, which implies $\mu(x, y)=0$. So, $d(x, y)=0$ or $d(y, T(x))=0$.

If $d(x, y)=0$, then $x=y$.

If $d(y, T(x))=0$, then $y=T(x)$ but $x=T(y)$ and $T$ is continuous. So, $x=y$.

Thus, $T$ has a fixed point in $X$.

Corollary 3.7: If the map $T$ in the theorems (3.3-3.6) be bijective, then $T$ has a unique fixed point.

Example 3.8: Let us consider the digital metric space $(X, d, \rho)$, with the digital metric defined by $d(x, y)=$ $|x-y|$. Consider the self mapping $T: X \rightarrow X$ given by, $T(x)=2 x+1$. Clearly, $T$ is an expansive mapping. Now, for every $y \in X$, there exists an $x=\frac{y-1}{2} \in X$, such that, $y=T(x)$. So, $T$ is onto. So, it satisfies the axioms of our theorem 3.2. Clearly, its fixed point is $x=-1$.

Example 3.9: Let us consider the digital metric space $(X, d, \rho)$, with the digital metric defined by $d(x, y)=$ $|x-y|$. Consider the self mapping $T: X \rightarrow X$ given by,

$$
T(x)=\left\{\begin{array}{ll}
2 x & \text { for } x<1 \\
2 x-1 & \text { for } x \geq 1
\end{array} .\right.
$$

Clearly, $T$ satisfies the condition

$$
d(T(x), T(y)) \geq k\{d(x, T(x))+d(y, T(y))\} .
$$

Now, for every $y \in X$, there exists an $x=\left\{\begin{array}{ll}\frac{y}{2} & \text { for } y<2 \\ \frac{y+1}{2} & \text { for } y \geq 2\end{array} \in X\right.$, such that, $y=T(x)$. So, $T$ is onto. So, it satisfies the axioms of our theorem 3.3. Clearly, it has two fixed points $x=0$ and $x=1$.

\subsection{Digital- $\alpha-\psi$-expansive Maps}

We introduce the concept of digital- $\alpha-\psi$-expansive mappings in non Newtonian metric spaces as follows:

Definition 3.10: Let $(X, d, \rho)$ be a digital metric space and $T: X \rightarrow X$ be a given mapping. We say that $T$ is a digital$\alpha-\psi$-expansive mapping, if there exists two functions $\alpha: X \times X \rightarrow[0, \infty)$ and $\psi \in \Psi$ such that for all $x, y$ in $X$, we have 


$$
\psi(d(T x, T y)) \geq \alpha(x, y) d(x, y) .
$$

Remark 3.11: Clearly, any expansive mapping is a digital- $\alpha$ - $\psi$-expansive mapping with $\alpha(x, y)=1$ for all $x, y \in X$ and $\psi(t)=k t$, for all $t \geq 0$ and $k \in(0,1)$.

Definition 3.12: Let $T: X \rightarrow X$ and $\alpha: X \times X \rightarrow$ $[0, \infty)$. We say that $T$ is $\alpha$-admissible if for all $x, y$ in $X$, we have $\alpha(x, y) \geq 1 \Rightarrow \alpha(T x, T y) \geq 1$.

Now, we prove our main results.

Theorem 3.13: Let $(X, d, \rho)$ be a complete digital metric space and $T: X \rightarrow X$ be a bijective, digital- $\alpha-\psi-$-expansive mapping and satisfies the following conditions:

(i) $T^{-1}$ is $\alpha$-admissible;

(ii) There exists $x_{0} \in X$ such that $\alpha\left(x_{0}, T^{-1} x_{0}\right) \geq 1$;

(iii) $T$ is digitally continuous.

Then $T$ has a fixed point, that is, there exists $u$ in $X$ such that $T u=u$.

Proof. Let us define the sequence $\left\{x_{n}\right\}$ in $X$ by $x_{n}=$ $T x_{n+1}$ for all $n \geq 0$, where $x_{0} \in X$ is such that $\alpha\left(x_{0}, T^{-1} x_{0}\right) \geq 1$. If $x_{n}=x_{n+1}$ for some $n$, then $x_{n}$ is a fixed point of $T$. So, we can assume that $x_{n} \neq x_{n+1}$ for all $n$.

It is given that

$$
\alpha\left(x_{0}, x_{1}\right)=\alpha\left(x_{0}, T^{-1} x_{0}\right) \geq 1 .
$$

Recalling that $T^{-1}$ is $\alpha$-admissible, we have, $\alpha\left(T^{-1} x_{0}, T^{-1} x_{1}\right)=\alpha\left(x_{1}, x_{2}\right) \geq 1$.

Using mathematical induction, we obtain

$$
\alpha\left(x_{n}, x_{n+1}\right) \geq 1 \text { for all } n=0,1,2, \ldots
$$

From (1) and (2), it follows that for all $n \geq 1$, we have

$$
\begin{aligned}
& d\left(x_{n}, x_{n+1}\right) \leq \alpha\left(x_{n}, x_{n+1}\right) d\left(x_{n}, x_{n+1}\right) \\
& \leq \psi\left(d\left(T x_{n}, T x_{n+1}\right)\right)=\psi\left(d\left(x_{n-1}, x_{n}\right)\right) .
\end{aligned}
$$

Since $\psi$ is non-decreasing, by induction, we have

$$
d\left(x_{n}, x_{n+1}\right) \leq \psi^{n}\left(d\left(x_{n-1}, x_{n}\right) \text { for all } n \geq 1 .\right.
$$

Using (3), we have

$$
\begin{aligned}
& d\left(x_{n}, x_{m}\right) \\
& \leq d\left(x_{n}, x_{n+1}\right)+d\left(x_{n+1}, x_{n+2}\right)+\ldots+d\left(x_{m-1}, x_{m}\right) \\
& \leq \sum_{k=n}^{m-1} d\left(x_{k}, x_{k+1}\right) \leq \sum_{k=n}^{m-1} \psi^{k}\left(d\left(x_{0}, x_{1}\right)\right) .
\end{aligned}
$$

Since, $\quad \psi \in \Psi$ and $d\left(x_{0}, x_{1}\right)>0$, we $\sum_{k=0}^{\infty} \psi^{k}\left(d\left(x_{0}, x_{1}\right)\right)<\infty$.

Thus, we have $\lim _{n \rightarrow \infty} d\left(x_{n}, x_{m}\right)=0$.

This implies that $\left\{x_{n}\right\}$ is a digital Cauchy sequence in digital metric space $(X, d, \kappa)$. But $(X, d, \kappa)$ is complete, so there exists $u$ in $X$ such that $x_{n} \rightarrow u$ as $n \rightarrow \infty$. From the continuity of $T$, it follows that $x_{n}=T x_{n+1} \rightarrow T u$ as $n \rightarrow \infty$. By the uniqueness of the limit, we get $u=T u$, that is, $u$ is a fixed point of $T$.

In what follows, we prove that Theorem 3.13 still holds for $T$ not necessarily continuous, assuming the following condition:

(M) If $\left\{x_{n}\right\}$ is a sequence in $X$ such that $\alpha\left(x_{n}, x_{n+1}\right) \geq$ 1 for all $n$ and $\left\{x_{n}\right\} \rightarrow x \in X$ as $n \rightarrow \infty$, then

$$
\alpha\left(T^{-1} x, T^{-1} x_{n}\right) \geq 1 \text {, for all } n \text {. }
$$

Theorem 3.14: If in Theorem 3.13, we replace the continuity of $T$ by the condition (M), then the result holds true.

Proof. Following the proof of Theorem 3.11, we know that $\left\{x_{n}\right\}$ is a digital Cauchy sequence in $X$ such that $\alpha\left(x_{n}, x_{n+1}\right) \geq 1$ for all $n$ and $x_{n} \rightarrow u$ as $n \rightarrow \infty$. Now, from the hypothesis (4), we have

$$
\alpha\left(T^{-1} u, T^{-1} x_{n}\right) \geq 1 \text {, for all } n \text {. }
$$

Using (1) and (5), we get

$$
\begin{aligned}
& d\left(T^{-1} u, u\right) \leq d\left(T^{-1} u, x_{n+1}\right)+d\left(x_{n+1}, u\right) \\
& =d\left(T^{-1} u, T^{-1} x_{n}\right)+d\left(x_{n+1}, u\right) \\
& \leq \alpha\left(T^{-1} u, T^{-1} x_{n}\right) d\left(T^{-1} u, x_{n+1}\right)+d\left(x_{n+1}, u\right) \\
& \leq \psi\left(d\left(u, x_{n}\right)\right)+d\left(x_{n+1}, u\right) .
\end{aligned}
$$

Continuity of $\psi$ at $t=0$ implies that $d\left(T^{-1} u, u\right)=0$ as $n \rightarrow \infty$. That is, $T^{-1} u=u$. Consider, $T u=$ $T\left(T^{-1} u\right)=u$, which implies that, $u$ is a fixed point of $T$.

We now present some examples in support of our results and show that the hypotheses in Theorems 3.11 and 3.12 do not guarantee uniqueness.

To ensure the uniqueness of the fixed point in Theorems 3.11 and 3.12, we consider the condition:

(S): For all $u, v \in X$, there exists $w \in X$ such that $\alpha(u, w) \geq 1$ and $\alpha(v, w) \geq 1$.

Theorem 3.15: Adding the condition (S) to the hypotheses of Theorem 3.13 (resp. Theorem 3.14), we obtain the uniqueness of the fixed point of $T$.

Proof. From Theorem 3.13 and 3.14, the set of fixed points is non-empty. We shall show that if $u$ and $v$ are two fixed points of $T$, that is, $T(u)=u$ and $T(v)=v$, then $u=v$.

From the condition (S), there exists $w \in X$ such that

$$
\alpha(u, w) \geq 1 \text { and } \alpha(v, w, w) \geq 1 .
$$

Recalling the $\alpha$-admissible property of $T^{-1}$, we obtain from (6)

$$
\alpha\left(u, T^{-1} w\right) \geq 1 \text { and } \alpha\left(v, T^{-1} w\right) \geq 1 .
$$

Therefore, by repeatedly applying the $\alpha$-admissible property of $T^{-1}$, we get

$$
\alpha\left(u, T^{-n} w\right) \geq 1 \text { and } \alpha\left(v, T^{-n} w\right) \geq 1 \text {, for all } n \text { in } \mathbb{N} \text {. }
$$

From the inequalities (1) and (8), we get

$$
\begin{aligned}
& d\left(u, T^{-n} w\right) \leq \alpha\left(u, T^{-n} w\right) d\left(u, T^{-n} w\right) \\
& \leq \psi\left(d\left(T u, T^{-n+1} w\right)=\psi\left(d\left(u, T^{-n+1} w\right)\right) .\right.
\end{aligned}
$$

Repetition of the above inequality implies that $d\left(u, T^{-n} w\right) \leq \psi(d(u, w))$, for all $n \in \mathbb{N}$. Thus, we have $T^{-n} w \rightarrow u$ as $n \rightarrow \infty$.

Using the similar steps as above, we obtain $T^{-n} w \rightarrow v$ as $n \rightarrow \infty$. Now, the uniqueness of the limit of $T^{-n} w$ gives $u=v$. This completes the proof.

Example 3.16: Let us consider the digital metric space $(X, d, \rho)$, with the digital metric defined by $d(x, y)=$ $|x-y|$. Consider the self mapping $T: X \rightarrow X$ given by

$$
T(x)= \begin{cases}2 x-\frac{11}{6}, & \text { for } x>1 \\ \frac{x}{6}, & \text { for } x \leq 1\end{cases}
$$


and $\alpha: X \times X \rightarrow[0, \infty)$ by

$$
\alpha(x, y)=\left\{\begin{array}{l}
0 \text { if } x, y \in[0,1) \\
1 \text { otherwise }
\end{array} .\right.
$$

Clearly, $T$ is a digital- $\alpha-\psi$-expansive mapping with $\psi(a)=\frac{a}{6}$ for all $a \geq 0$. In fact for all $x, y \in X$, we have $d(T x, T y) \geq \alpha(x, y) d(x, y)$.

Moreover, there exists $x_{0} \in X$ such that $\alpha\left(x_{0}, T^{-1} x_{0}\right) \geq 1$. In fact, for $x=1$, we have $\alpha\left(1, T^{-1} 1\right)=1$. Obviously, $T$ is non Newtonian continuous, and so it remains to show that $T^{-1}$ is $\alpha$-admissible. For this, let $x, y \in X$ such that $\alpha(x, y) \geq 1$. This implies that $x \geq 1$ and $y \geq 1$, and by the definitions of $T^{-1}$ and $\alpha$, we have $T^{-1}(x)=\frac{x}{2}+\frac{11}{12} \geq 1, T^{-1}(y)=$ $\frac{y}{2}+\frac{11}{12} \geq 1$ and $\alpha\left(T^{-1} x, T^{-1} y\right)=1$. Then $T^{-1}$ is $\alpha$-admissible. Now, all the hypothesis of Theorem 3.13 are satisfied. Consequently, $T$ has a fixed point. Clearly, $x=0$ and $x=\frac{11}{6}$ are two fixed points of $T$.

Now, we give an example involving a function $T$ that is digitally discontinuous.

Example 3.17: Let us consider the digital metric space $(X, d, \rho)$, with the digital metric defined by $d(x, y)=$ $|x-y|$. Consider the self mapping $T: X \rightarrow X$ given by

$$
T(x)= \begin{cases}\frac{x}{2}-\frac{5}{2} & \text { for } x>1 \\ \frac{x}{5} & \text { for } x \leq 1\end{cases}
$$

and $\alpha: X \times X \rightarrow[0, \infty)$ by

$$
\alpha(x, y)=\left\{\begin{array}{l}
0 \text { if } x, y \in[0,1) \\
1 \text { otherwise }
\end{array} .\right.
$$

Due to the discontinuity of $T$ at 1 , Theorem 3.14 is not applicable in this case. Clearly, $T$ is a digital- $\alpha-\psi-$ expansive mapping with $\psi(a)=\frac{a}{5}$ for all $a \geq 0$. In fact for all $x, y$ in $X$, we have $\frac{1}{5} d(T x, T y) \geq \beta(x, y) d(x, y)$. Moreover, there exists $x_{0} \in X$ such that $\alpha\left(x_{0}, T^{-1} x_{0}\right) \geq 1$. In fact, for $x_{0}=1$, we have $\alpha\left(1, T^{-1} 1\right)=1$. Now, let $x, y \in X$ such that $\alpha(x, y) \geq 1$. This implies that $x \geq 1$ and $y \geq 1$, and by the definitions of $T^{-1}$ and $\alpha$, we have $T^{-1}(x)=\frac{x}{2}+\frac{5}{4} \geq 1, \quad T^{-1}(y)=\frac{y}{2}+\frac{5}{4} \geq 1$, and $\alpha\left(T^{-1} x, T^{-1} y\right)=1$. Then $T^{-1}$ is $\alpha$-admissible. Finally, let $\left\{x_{n}\right\}$ be a sequence in $X$ such that $\alpha\left(x_{n}, x_{n+1}\right) \geq 1$ for all $n$ and $\left\{x_{n}\right\} \rightarrow x \in X$ as $n \rightarrow \infty$. Since $\alpha\left(x_{n}, x_{n+1}\right) \geq 1$ for all $n$, by the definition of $\alpha$, we have $\alpha\left(T^{-1} x, T^{-1} x_{n}\right)=1$. Therefore, all the required hypothesis of Theorem 3.14 are satisfied and so $T$ has a fixed point. Here, $x=0$ and $x=\frac{5}{2}$ are two fixed points of $T$.

\subsection{Application of Expansive Mappings to Digital Images}

The zooming in and zooming out of the digital images may be very important in certain circumstances. But when we zoom out an image due to the use of pixels the image quality is distorted and the image becomes blurred. By assigning an expansive map to the points of the image the procedure may become more fruitful, i.e., the image can be zoomed out without disturbing the quality of the image.

\subsection{Scope of the Study}

The aim of this paper is to give the digital version of some fixed point theorems where the map is expansive. We hope the results will be useful in digital topology and fixed point theory. These results can therefore be used for the expansion or Zooming out of digital images and the array of digital images. In future, some other properties of digital images can be discussed with the viewpoint of fixed point theory.

\section{Competing Interests}

The Authors declare that they have no competing interest.

\section{References}

[1] L. E. S. Brouwer, Uber Abbildungen Von Mannigfaltigkeiten, Math. Ann., 77(1912), 97-115.

[2] L. Boxer, Digitally Continuous Functions, Pattern Recognition Letters, 15 (1994), 833-839.

[3] L. Boxer, Properties of Digital Homotopy, J. Math. Imaging Vis., 22(2005), 19-26.

[4] L. Boxer, A Classical Constructions for The Digital Fundamental Group, J. Math. Imaging Vis., 10(1999), 51-62.

[5] L. Boxer, Digital Products, Wedges and Covering Spaces, J. Math. Imaging Vis., 25(2006), 159-171.

[6] L. Boxer, Continuous Maps on Digital Simple Closed Curves, Appl. Math., 1(2010), 377-386.

[7] L. Boxer, O. Ege, I. Karaca, J. Lopez and J. Louwsma, Digital fixed points, approximate fixed points, and universal functions, Applied General Topology, 17(2), 159-172 (2016).

[8] O. Ege, I. Karaca, Fundamental Properties of Simplicial Homology Groups for Digital Images, American Journal of Computer Technology and Application, 1(2013), 25-42.

[9] O. Ege, I. Karaca, Lefschetz Fixed Point Theorem for Digital Images, Fixed Point Theory Appl., 2013(2013), 13 pages.

[10] O. Ege, I. Karaca, Applications of The Lefschetz Number to Digital Images, Bull. Belg. Math. Soc. Simon Stevin, 21(2014), 823-839.

[11] O. Ege, I.karaca, Banach Fixed Point Theorem for Digital Images, J. Nonlinear Sci. Appl., 8(2015), 237-245.

[12] O. Ege and I. Karaca, Digital homotopy fixed point theory, Comptes Rendus Mathematique, 353(11), 1029-1033 (2015).

[13] O. Ege, Complex valued Gb -metric spaces, Journal of Computational Analysis and Applications, 21(2), 363-368 (2016).

[14] O. Ege and I. Karaca, Digital fibrations, Proceedings of the National Academy of Sciences, India Section A: Physical Sciences, 87(1), 109-114 (2017).

[15] O. Ege and I. Karaca, Nielsen fixed point theory for digital images, Journal of 2 Computational Analysis and Applications, 22(5), 874880 (2017).

[16] G. T. Herman, Oriented Surfaces in Digital Spaces, CVGIP: Graphical Models and Image Processing, 55(1993), 381-396. 
[17] I. Karaca, O. Ege, Some Results on Simplicial Homology Groups of 2D Digital Images, Int. J. Inform. Computer Sci., 1(2012), 198-203.

[18] T. Y. Kong, A Digital Fundamental Group, Computers and Graphics, 13(1989), 159-166.

[19] A. Rosenfeld, Digital Topology, Amer. Math. Monthly, 86(1979), 76-87.
[20] B. Samet, C. Vetro, P. Vetro, Fixed point theorem for $\alpha-\psi$-contractive type mappings, Nonlinear Analysis 75 (2012), 2154-2165.

[21] S. Z. Wang, B. Y. Li, Z. M. Gao, K. Iseki, Some fixed point theorems on expansion mappings, Math. Jpn. 29, 631-636, 1984. 